Time-Varying Systems and Computations 


\section{Time-Varying Systems and Computations}

by

PATRICK DEWILDE

and

ALLE-JAN VAN DER VEEN

DIMES, Delft University of Technology,

Delft, The Netherlands

SPRINGER-SCIENCE+BUSINESS MEDIA, B.V. 
Library of Congress Cataloging-in-Publication Data

ISBN 978-1-4419-5045-1 ISBN 978-1-4757-2817-0 (eBook)

DOI $10.1007 / 978-1-4757-2817-0$

Printed on acid-free paper
All Rights Reserved
(C) 1998 Springer Science+Business Media Dordrecht
Originally published by Kluwer Academic Publishers in 1998
Softcover reprint of the hardcover 1st edition 1998
No part of the material protected by this copyright notice may be reproduced or utilized in any form or by any means, electronic or mechanical, including photocopying, recording or by any information storage and retrieval system, without written permission from the copyright owner. 


\section{Contents}

Preface $\quad$ ix

Acknowledgments $\quad$ xiii

1. INTRODUCTION 1

1.1 Computational linear algebra and time-varying systems 1

$\begin{array}{lll}1.2 & \text { Objectives of computational modeling } & 7\end{array}$

$\begin{array}{ll}1.3 & \text { Connections and alternative approaches } \\ \end{array}$

\section{Part I REALIZATION}

2. NOTATION AND PROPERTIES OF NON-UNIFORM SPACES 19

$\begin{array}{ll}2.1 \text { Spaces of non-uniform dimensions } & 20\end{array}$

2.2 Shifts and diagonal representations 26

$\begin{array}{ll}2.3 \text { Notes } & 30\end{array}$

3. TIME-VARYING STATE SPACE REALIZATIONS 33

3.1 Realizations of a transfer operator 34

3.2 Special classes of time-varying systems 41

3.3 Examples and extensions 46

3.4 Realization theory for finite matrices 52

$\begin{array}{ll}3.5 & \text { Identification from input-output data } \\ 3.6 & 62\end{array}$

3.6 Realization theory for matrices of low displacement rank 65

4. DIAGONAL ALGEBRA 73

$\begin{array}{ll}4.1 \text { Sequences of diagonals } & 74\end{array}$

4.2 The diagonal algebra of $\mathcal{X}_{2} \quad 76$

$\begin{array}{ll}4.3 \text { Sliced bases and projections in } \mathcal{X}_{2} & 79\end{array}$

5. OPERATOR REALIZATION THEORY 87

5.1 The Hankel operator 88

$\begin{array}{lll}5.2 & \text { Reachability and observability operators } & 91\end{array}$

5.3 Reachability and observability Gramians 95 
$\begin{array}{lll}5.4 & \text { Abstract realization theory } & 102\end{array}$

$\begin{array}{ll}5.5 \text { Notes } & 116\end{array}$

6. ISOMETRIC AND INNER OPERATORS 121

6.1 Realization of inner operators 122

6.2 External factorization 126

6.3 State-space properties of isometric systems 132

6.4 Beurling-Lax like theorem 136

6.5 Example 142

7. INNER-OUTER FACTORIZATION AND OPERATOR INVERSION 145

$\begin{array}{ll}7.1 \text { Introduction } & 146\end{array}$

\begin{tabular}{ll}
7.2 & Inner-outer factorizations \\
\hline
\end{tabular}

$\begin{array}{lll}7.3 & \text { Operator inversion } & 165\end{array}$

$\begin{array}{lll}7.4 & \text { Examples } & 172\end{array}$

7.5 Zero structure and its limiting behavior 179

$\begin{array}{lll}7.6 & \text { Notes } & 186\end{array}$

Part II INTERPOLATION AND APPROXIMATION

8. J-UNITARY OPERATORS 191

$\begin{array}{ll}8.1 \text { Scattering operators } & 191\end{array}$

8.2 Geometry of diagonal J-inner product spaces 200

8.3 State space properties of J-unitary operators 204

$\begin{array}{lll}8.4 & \text { Past and future scattering operators } & 210\end{array}$

$\begin{array}{lll}8.5 & \text { J-unitary external factorization } & 215\end{array}$

8.6 J-lossless and J-inner chain scattering operators 218

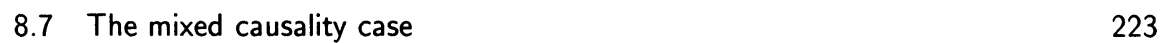

9. ALGEBRAIC INTERPOLATION 233

$\begin{array}{ll}9.1 \text { Diagonal evaluations or the W-transform } & 235\end{array}$

9.2 The algebraic Nevanlinna-Pick problem 237

9.3 The tangential Nevanlinna-Pick problem 242

9.4 The Hermite-Fejer interpolation problem 242

9.5 Conjugation of a left interpolation problem 245

$\begin{array}{ll}9.6 & \text { Two sided interpolation } \\ 9.7 & 250\end{array}$

$\begin{array}{ll}9.7 \text { The four block problem } & 260\end{array}$

10. HANKEL-NORM MODEL REDUCTION 263

$\begin{array}{ll}10.1 \text { Introduction } & 264\end{array}$

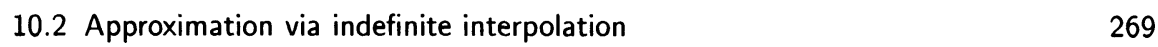

10.3 State realization of the approximant 276

10.4 Parametrization of all approximants $\quad 282$

$\begin{array}{ll}10.5 \text { Schur-type recursive interpolation } & 292\end{array}$ 
$\begin{array}{ll}\text { 10.6 The Nehari problem } & 300\end{array}$

$\begin{array}{ll}10.7 \text { Concluding remarks } & 306\end{array}$

11. LOW-RANK MATRIX APPROXIMATION AND SUBSPACE TRACKING 307

$\begin{array}{ll}11.1 \text { Introduction } & 307\end{array}$

11.2 J-unitary matrices $\quad 309$

$\begin{array}{ll}11.3 \text { Approximation theory } & 311\end{array}$

$\begin{array}{ll}11.4 \text { Hyperbolic QR factorization } & 316\end{array}$

11.5 Hyperbolic URV decomposition $\quad 322$

11.6 Updating the SSE-2 325

11.7 Notes 331

Part III FACTORIZATION

12. ORTHOGONAL EMBEDDING 337

$\begin{array}{ll}12.1 \text { Introduction and connections } & 338\end{array}$

$\begin{array}{ll}12.2 \text { Strictly contractive systems } & 343\end{array}$

$\begin{array}{ll}12.3 \text { Contractive systems: the boundary case } & 347\end{array}$

$\begin{array}{ll}12.4 \text { Lossless embedding } & 351\end{array}$

12.5 Numerical issues $\quad 354$

$\begin{array}{ll}12.6 \text { Notes } & 359\end{array}$

13. SPECTRAL FACTORIZATION 363

$\begin{array}{ll}13.1 \text { Introduction } & 363\end{array}$

$\begin{array}{ll}13.2 \text { Spectral factorization } & 365\end{array}$

$\begin{array}{ll}\text { 13.3 Computational issues } & 371\end{array}$

13.4 Convergence of the Riccati recursion 372

$\begin{array}{ll}13.5 \text { Connections } & 376\end{array}$

$\begin{array}{ll}13.6 \text { Notes } & 380\end{array}$

14. LOSSLESS CASCADE FACTORIZATIONS 383

14.1 Time-invariant cascade factorizations 383

14.2 Parsimonious parametrization of contractive LTI systems 390

14.3 Time-varying $\Sigma$-based cascade factorization 397

14.4 Time-varying $\Theta$-based cascade factorization $\quad 410$

15. CONCLUSION 419

Appendices $\quad 423$

$\begin{array}{ll}\text { A-Hilbert space definitions and properties } & 423\end{array}$

$\begin{array}{ll}\text { References } & 435\end{array}$

Glossary of notation $\quad 453$

Index $\quad 455$ 
Complex function theory and linear algebra provide much of the basic mathematics needed by engineers engaged in numerical computations, signal processing or control. The transfer function of a linear time invariant system is a function of the complex variable $s$ or $z$ and it is analytic in a large part of the complex plane. Many important properties of the system for which it is a transfer function are related to its analytic properties. On the other hand, engineers often encounter small and large matrices which describe (linear) maps between physically important quantities. In both cases similar mathematical and computational problems occur: operators, be they transfer functions or matrices, have to be simplified, approximated, decomposed and realized. Each field has developed theory and techniques to solve the main common problems encountered.

Yet, there is a large, mysterious gap between complex function theory and numerical linear algebra. For example, complex function theory has solved the problem to find analytic functions of minimal complexity and minimal supremum norm that approximate given values at strategic points in the complex plane. They serve e.g., as optimal approximants for a desired behavior of a system to be designed. No similar approximation theory for matrices existed until recently, except for the case where the matrix is (very) close to singular. The relevant approximation theory in the complex plane is spectacular and has found a manifold of applications such as broadband matching, minimal sensitivity control, and the solution of differential game problems. A similar "linear algebra" result would without doubt be very desirable. Over the years we have discovered that a strong link between the two theories can indeed be developed.

To establish this link, one has to move away from the classical idiosyncrasies of the two basic theories, and develop a new and somewhat unusual paradigm, which, however, turns out to be quite natural and practical once one gets used to it. Classical matrix theory and linear algebra act on vectors and matrices. Very early in the development of these theories it was found beneficial to move from single scalar quantities and variables to vector representations. This has been an important lift in the level of abstraction, with great importance for physics and engineering, and also largely motivated by them. It has allowed for compact, insightful algebraic notations which have been adopted by a large variety of fields in which multidimensional objects interact with each other. Mechanics, electromagnetism, quantum mechanics, operations re- 
search, electrical network theory and signal processing all are fields which have been deeply influenced by vector and matrix calculus.

With the advent of powerful electronic computing, global vector or matrix-vector operations may even be viewed as atomic numerical operations. A vector computer can be programmed to execute them in parallel by a single instruction. A matrix-vector or matrix-matrix multiplication, a matrix inversion, and more complicated operations such as the calculation of matrix eigenvalues, can easily be conceived as simple sequences of such massive vector operations. In this book we will add another basic, vector-like quantity to the arsenal of objects handled by linear algebra. The new object represents a diagonal of a matrix or an operator. Thus, in addition to matrix operations acting on rows or columns, we shall consider elementary operations on diagonals. These, in fact, can be handled with the same ease by a vector or parallel computer, but they will have a very different algebraic interpretation.

What is to be gained by such an approach? In the course of the book, we develop a forceful argument that indeed, it allows us to solve several problems in linear algebra whose solutions were either unknown, or obscured by the traditional approach. In addition, the theory also creates its own class of new problems. The missing theoretical link is provided by system theory, in our case the theory of linear, time discrete and time varying dynamical systems. For example, we look at the meaning of "computational complexity" from a system theoretical point of view. Up to now, classical linear algebra had only a limited notion of complexity, restricted to either matrices that are sparse (most entries equal to zero), or matrices that are close to singular. The sparse structure is easily destroyed by algebraic operations: even the inverse of such a matrix is not sparse, and as a result, it seems that multiplication by this inverse is a full-complexity operation. This does not happen with a system theoretic "realization": it is straightforward to show that a minimal realization of the inverse has the same complexity as one for the original. In addition, system theory will allow us to derive a powerful approximation theory that maps a given matrix to a matrix of lowest computational complexity (in the system theoretical sense), given a certain tolerance.

System theory has already had a significant impact on linear algebra, mostly in the study of Toeplitz matrices and similar structured matrix problems. These are connected to time-invariant systems. Our approach in this book is complementary: we generalize to time-varying systems, which allows to describe any matrix. The structure in the matrix we are looking for is now less obvious, it is connected to the rank of certain strategic submatrices. In the course of the book, several classical results in the theory of time-varying systems are recovered: e.g., we hit on the all-pervasive time-varying Riccati equation, the bounded real lemma and the related Kalman-Yakubovitch-Popov lemma. Still, we believe that we are placing the theory in the context of a new "paradigm", i.e., a realm of problems and solutions with their own dynamics. Indeed, several results in time-varying system theory that were only known as abstract theory (such as proofs by Arveson of the existence of inner-outer factorizations) have now become explicit "constructive operator theory". Significant new results in this context are the time-varying Hankel-norm approximation theory, as well as the solution of several interpolation problems, leading to a generalization of the minimal sensitivity problem and optimal control theory. 
An added value of the book is the very explicit link which it lays between numerical linear algebra and generalizations of analytic function theory on the open unit disc, as traditionally applied to transfer function calculus. The reader will discover the algebraic generalizations of a host of classical interpolation problems: Schur, NevanlinnaPick, Caratheodory-Fejer, Nehari, Schur-Takagi. These provide natural solutions to nice problems in algebra. Conversely, elementary methods in numerical analysis have interesting counterparts in system theory where their interpretation is non-trivial. E.g., we show that inner-outer factorization can be viewed as a generalization of $\mathrm{QR}$ factorization, and Hankel-norm model reduction can be used for efficient subspace estima tion and tracking.

We do not limit ourselves to finite matrices. The connection to system theory allows to derive meaningful results also for "infinite" matrices, or operators on a Hilbert space. From a linear algebra point of view, the results are perhaps uncanny: e.g., the inverse of an upper triangular infinite matrix need not be upper triangular! The connection to time-varying systems gives insight into the mechanics of this: the inverse of an upper operator is upper if and only if the original system has a property which is called "outer". Even for linear algebra, infinite linear systems are useful: such systems occur e.g., in the discretization of differential equations where the boundary condition is conveniently placed at infinity. Because the matrix entries become constant as we move away from the center of the matrix, it can still be described by a finite number of parameters. It has been amply demonstrated that such a procedure may lead to more accurate overall results. The downside of this generality is perhaps that, in order to obtain precise results, Hilbert space theory plays a major but sometimes also a mere technical role. (We summarize some basic notions of Hilbert space theory in an appendix.)

For whom is this book intended? We suggest the following.

- It can be used as a graduate course in linear time-varying system theory: all the main concepts and problems are there, and they are treated in a direct and streamlined manner. For this purpose we have been somewhat lengthy in developing the basic framework in the first chapters - our excuses to interested mathematicians!

- It can be used as a source of new problems in numerical linear algebra, with a concurrent new methodology to solve them. Several theories in the book scream for in-depth analysis, in particular the theory of optimal sensitivity reduction, the inversion theory for infinite systems of equations and the optimal matrix-operator approximation theory.

- It can also be used as an introductory course in a new topic: "theory of computational systems". Although the material presented here falls short of a comprehensive theory - the subject matter presently does not go far beyond linear problems and computations - we do think that there is already sufficient information to justify independent interest.

It is our hope that the algebraic system's community will find inspiration and motivation in the theory presented here. Although it has definite affinities to Arveson's "Nested Algebras" and Saeks and Feintuch's "Resolution Spaces", it does have a new flavor, mainly because of its direct link to numerical procedures via the treatment of diagonals as the new vectorial object. 


\section{Acknowledgments}

Our gratitude goes to many fellow scientists and colleagues with whom we have had fruitful interactions, and we hope that we have given due reference to their contributions in the various chapters of this book. We do wish to single out two colleagues and friends who have contributed directly and materially to the realization of this book, in a scientific sense and by providing for hospitality and the environment in which the redactional work could progress. Harry Dym and Tom Kailath have been generous hosts to both of us, several times and for extended periods. Both had a decisive influence on the theory, providing insights and contributing key steps. We had intense exchanges of ideas and information with many of their students as well, too many to justify the compilation of a long list. Furthermore, we wish to thank students, colleagues and supporting staff in Delft, for the cordial atmosphere in which we were allowed to function. If one person in Delft has been outstanding in assisting us in almost any way one can think of (except scientifically), it is Mrs. Corrie Boers. Finally, Patrick Dewilde wants to voice his gratitude to his charming wife Anne for the continuous and warm support she has provided over the years. 